Magnetic Separation News, 1985, Vol. pp. 183-184 0731-3632/85/0104-0183 \$25.00/0
(C) 1985 Gordon and Breach, Science Publishers, Inc. and OPA Ltd. Printed in the United Kingdom

\title{
forthcoming meetings
}

\author{
International Symposium-Workshop \\ on \\ Particulate and Multi-Phase Processes \\ Miami Beach 22-26 April 1985
}

For further information contact:

Ms. Sheila Puryear

Clean Energy Research Institute

University College of Miami,

P.0. Box 248294

Coral Gables, Florida 33124

U.S.A.

XVth International Mineral

Processing Congress

Cannes, 2-9 June, 1985

For further information contact:

R. Bloise

BP 6009

Orleans, Cedex, France. 


\section{CONFERENCE DIARY}

Conference

International Symposium

-Workshop on Particulate and Multi-Phase Processes

23rd International Magnetics Conference (Intermag)

15 th Internationa1 Minera1 Processing Congress

9 th International Conference on Magnet Technology
Organizing Body/ Organizer

Fine Particle Society.

I.E.E.E.

E.J. Torok, Sperry-Univac PO Box 3525 MS U2-P26

St. Paul, Mn 55165 U.S.A.

R. Bloise

BP 6009

Orleans,

Cedex, France.

P. Weymuth MT-9 Secretary SIN 5234 Villigen Switzerland.
Date and Location

22-26 April 1985, Miami Beach, Florida, U.S.A.

April 29 May 2, 1985, St. Paul, Mn U.S.A.

June 2-9, 1985 Cannes, France.

September 9-13, 1985, Zurich Switzerland. 